\title{
VSearch: Macintosh software for experiments in visual search
}

\author{
JAMES T. ENNS, ERIC P. OCHS, and RONALD A. RENSINK \\ University of British Columbia, Vancouver, British Columbia, Canada
}

\begin{abstract}
In this paper, we describe software that turns a Macintosh computer into an off-the-shelf tool for experiments on visual search. Our design goals included portability (between members of the Macintosh computer family and between various research settings), user-friendliness (equivalent to Macintosh programming standards), flexibility (to allow replication and extension of important experiments on visual search), and adaptability (very short design-to-data and data-toanalysis turnaround times). We describe how the software meets these goals in three major phases of an experiment: stimulus construction, experimental control, and statistical analysis. We then list several landmark studies of visual search that can be easily designed and extended with the software. Finally, we outline plans for expanding the experimental variations that will be supported in future versions of the software.
\end{abstract}

The visual search task is a widely used research tool in the study of human vision. Experiments on visual search have been instrumental in shaping theories of early vision (Beck, 1982; Julesz, 1981; Zucker, 1987), attention (Neisser, 1967; Schneider \& Shiffrin, 1977; Treisman \& Gelade, 1980), reading (Gibson \& Levin, 1975), and computational vision (Tsotsos, 1988).

The essential elements of a visual search task are straightforward. The subject looks for a target item among a background of distractor items and reports the target's presence or absence. The researcher measures the time required to make this report and tries to understand the variables that influence search rate and accuracy. These variables include stimulus properties (e.g., target complexity and similarity) and observer characteristics (e.g., strategies, individual differences). In this paper, we describe two pieces of software, VSEARCH and IMAKER, that assist the vision researcher in the design, construction, and analysis of visual search experiments.

Macintosh computers are inexpensive and widely used, and they have sophisticated graphics capabilities. VSEARCH allows any of the Macintosh computers currently available to be used off-the-shelf as complete research stations for visual search. It thus eliminates the need for the specialized materials and equipment that researchers have had to use previously. Graphic art tools, supplies, and services are no longer needed for stimulus construction; neither electronic nor mechanical tachistoscopes are necessary for stimulus presentation; and it is no longer necessary to have computers devoted solely to data analysis. Our software uses the Macintosh to assist in each of these steps.

Correspondence may be addressed to James T. Enns, Department of Psychology, University of British Columbia, Vancouver, British Columbia V6T 1Y7, Canada.
IMAKER allows any image that can be drawn or imported to one of the popular Macintosh graphics applications (e.g., MacPaint or FullPaint) to be used in visual search experiments. Images are copied from the graphics application to the Macintosh clipboard (an operating system memory buffer accessible by any Macintosh application). Once in the clipboard, IMAKER can be used to create a file of the image, which can be accessed by VSEARCH.

Our description of the software is divided into four sections. In the first section, we outline the objectives that guided the development of the software. The second section treats how the software functions as a research tool in each stage of the experimental process. In the third section, we show how the software can be used to replicate and extend landmark findings in visual search. Finally, we discuss progress on improvements to the software that will permit a wider range of experimental control and design in future versions.

\section{Design Objectives for VSEARCH and IMAKER}

Portability. One of our foremost design goals was to use the Macintosh in an off-the-shelf mode. We believed this would allow us to create a research station that was portable in two senses of the word. First, we wanted to be able to run experiments on any member of the growing family of Macintosh computers (i.e., the $512 \mathrm{~K}$, Plus, SE, SE/30, II, and IIx). This would make it possible, for example, to design an experiment on one computer, collect data on another, and analyze data on still another. However, in addition to being able to move the software between machines, we wanted the research station itself to be transportable between various settings, such as university labs, schools, hospitals, and homes. Therefore, we looked for response input and timing solutions that did not depend on additional hardware. A detailed description of how precision response timing (millisecond ac- 
curacy) and precision display timing (tick, or $16.67-\mathrm{msec}$ accuracy) can be accomplished with an off-the-shelf Macintosh is given by one of us in the present issue of Behavior Research Methods, Instruments, \& Computers (Rensink, 1990).

Ease of use. Macintosh computers are known for their user friendliness, to the extent that they have raised the industry standard for human factors in software use. There were clear advantages to our following these standards. For example, it would permit the use of our software by undergraduates in experimental psychology teaching labs, as well as by research assistants without computer experience. Also, it would reduce the proportion of time and resources that vision researchers spend on stimulus preparation and experimental control. Thus, with the exception of typing in names for files that are created, all choices in VSEARCH are made by moving and clicking the mouse.

Adaptive Experimental Design. One of the greatest obstacles in the creation of new experiments is the large amount of time spent redrawing stimuli and rebuilding apparati in response to relatively minor changes in procedures or questions. Therefore, we strove for very rapid design-to-data and data-to-analysis turnaround times.

Design-to-data time was minimized by the creation of object-based rather than screen-based displays. That is, rather than have the user design full-screen images (i.e., slides) that contain examples of the target and distractor items, our software requires the user only to design target and distractor items. These items are then presented randomly on the screen, within the additional constraints that are specified by the user. A second feature that minimizes the design-to-data time is the use of existing graphics software for the construction of search objects. The software then uses an interface, IMAKER, to import digitized, line-based, hand-drawn, or any other images from existing graphics software.

Data-to-analysis time was minimized in a similar way by relying on commercially available statistical software for data analysis and graphing capabilities. VSEARCH stores data to files that can be read by many statistical programs.

Flexibility. We wanted the software to be flexible enough to replicate the paradigmatic findings in visual search. Also, we wanted to be able to extend previously used variations on the paradigm to novel domains and questions. In the next two sections, we describe the variations supported in the current version.

\section{Experimental Design and Data Analysis with VSEARCH and IMAKER}

VSEARCH is used in the three major phases of a visual search experiment as follows:

Stimulus construction. Most graphics editors available for the Macintosh support the importing and exporting of images through the clipboard. IMAKER will take any image in the clipboard and turn it into a file that can be used by VSEARCH. It can also be run in one of two modes. Used as a desk accessory (i.e., an operatıngsystem-resident program that can be run concurrently with any application), it permits the rapid conversion of graphics images into VSEARCH-readable files. Used as an application, it permits the rapid inspection of previously created IMAKER files.

Experimental control. VSEARCH allows the user to provide information concerning a large number of design questions, which are implemented in an environment that functions as an automated tachistoscope. Alternatively, VSEARCH contains default values for each of the design questions, thus permitting the program to run with minimal input from the user. (At the very minimum, a user must specify one target and one distractor item.) In either case, the user can adjust the procedural, display, and temporal parameters until the user is satisfied with the look and feel of the experiment.

Figure 1 shows the highest level menus in the program. Experimental design decisions (under the Setup menu) have been divided into menus that concern procedural issues (Experiment; Figure 2), display characteristics (Size Specifications; Figure 3), temporal parameters (Duration Specifications; Figure 4), and target and distractor images (Item Specifications; Figure 5).

Statistical analysis. There are many statistical packages available for the Macintosh. Most of these support the importing and exporting of data through the clipboard as well as through text-only files (e.g., Staview, MacSS, CLR Anova). VSEARCH creates text-only files that can be read by these packages.

The subject's response time and accuracy data are stored in a highly compact form to save disk space (the data for each response are represented in one integer). VSEARCH uses the stored experimental parameters and trial integers to regenerate all the data when asked to do so. There are three main ways in which the data are retrieved. First,

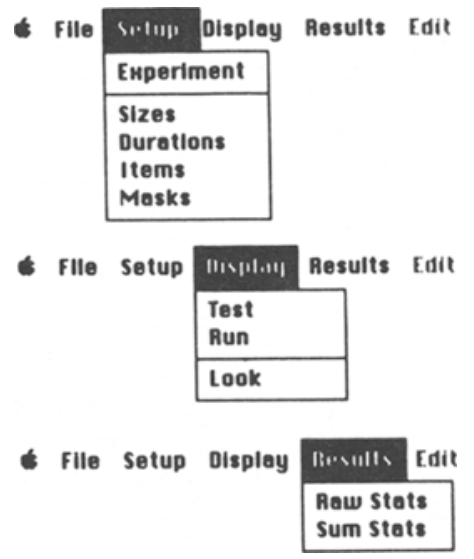

Figure 1. The highest level menus in VSearch. Experimental design decisions are made under the Setup menu. Displays may be tested (no data saved), run (data saved), or simply looked at under the Display menu. Under the Results menu, data may be examined, printed, or saved to disk in Raw or Summary form. 
at the end of each experimental session, summary data are displayed on the screen for each condition (i.e., mean response times, percentage accuracy, and response time slopes). Second, summary data may be displayed on the screen, printed out, and/or saved as text-only files at any time following data collection. Third, raw data files may be printed or saved as text-only files at any time. Figures 6 and 7 show the menus used to manipulate the summary data and raw data, respectively.

\section{Landmark Experiments in Visual Search}

The following is an incomplete list of experiments in visual search that have had a strong influence on theories of early vision and visual attention; they are representative of experiments that are easily designed and run with VSEARCH:

Letter search (Neisser, 1963, 1964). In one of the first systematic studies of visual search, Neisser demonstrated that the search rate for a target letter was a linear function of the total number of letters that were displayed.

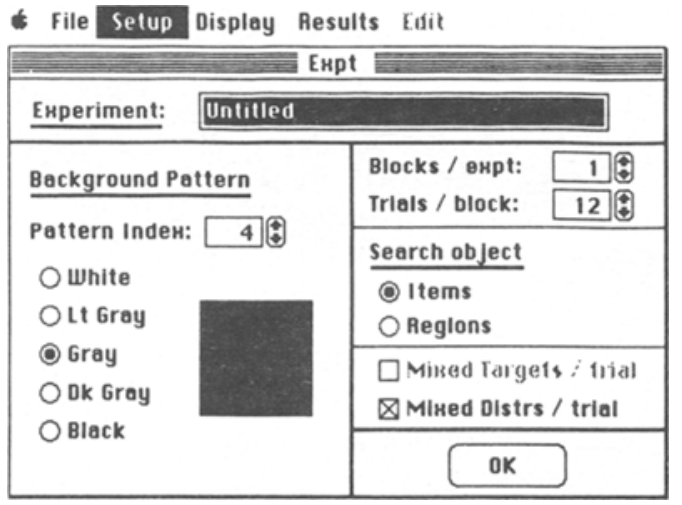

Figure 2. Decisions concerning the background pattern, the type of search object (item or region), the number of trials per block, and the number of blocks per experiment, are made in the Experiment menu.

* Flle Setup Display Results Edit

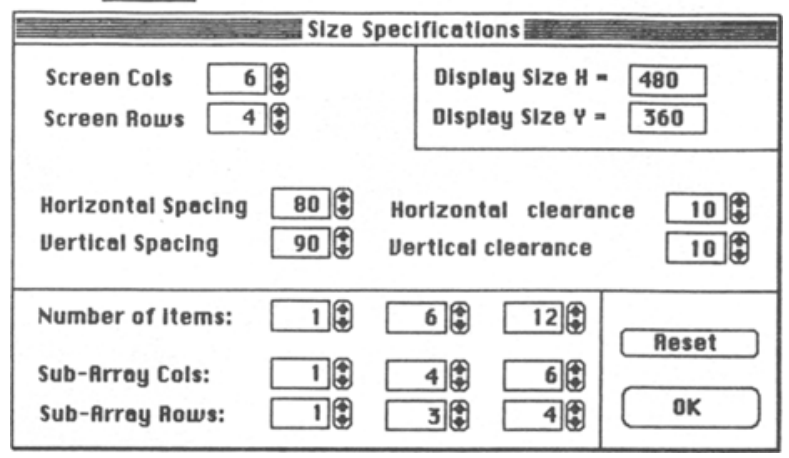

Figure 3. Display characteristics are specified in the Sizes menu. The screen is organized into virtual rows and columns. Items are placed randomly into the row by column locations, within the constraints of spacing and display size that are specified in this menu.

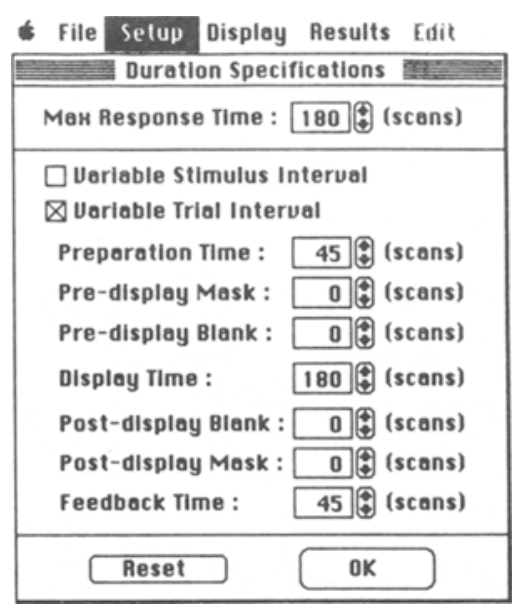

Figure 4. Temporal parameters are specified in units of video refresh cycles, or scans. Any given trial event can be further divided into eight different interval durations using the Variable Stimulus Interval mode.

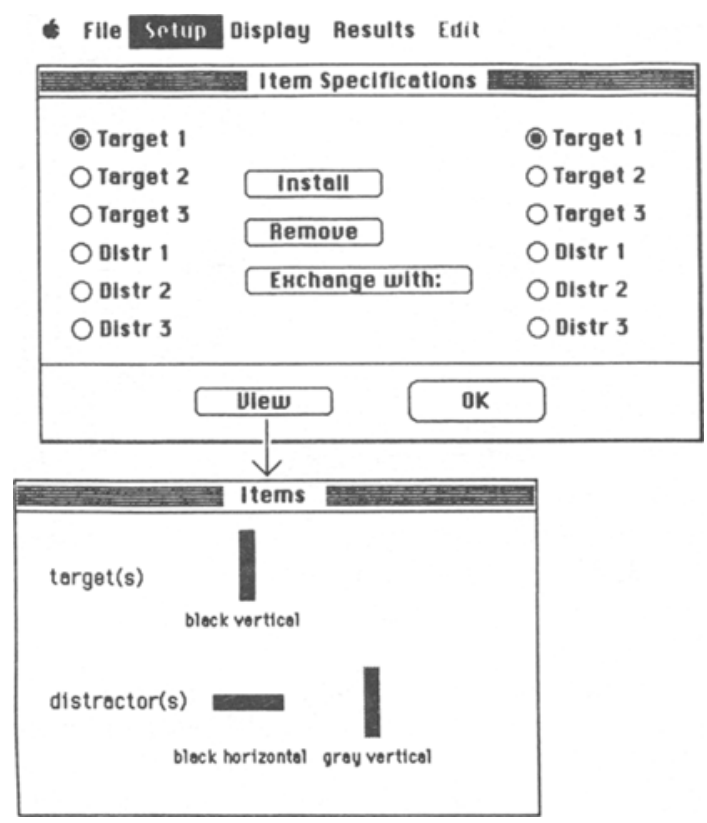

Figure 5. Up to three different target and distractor images may be installed and viewed, using the Item menu. The roles of target and distractor items are easily switched, using the Exchange with function.

A second factor that strongly influenced search rate was the similarity between target and distractor items (e.g., curved distractor letters allowed for more rapid search of $Z$ than straight-line distractor letters did). Neisser was also somewhat surprised to find that search for multiple targets (e.g., $\mathrm{K}$ or $\mathrm{Z}$ ) was often no slower than search for single targets (e.g., K).

Simple feature search (Treisman \& Gelade, 1980). Some targets can be reported as rapidly among few distractors as among many distractors (e.g., | versus _). 


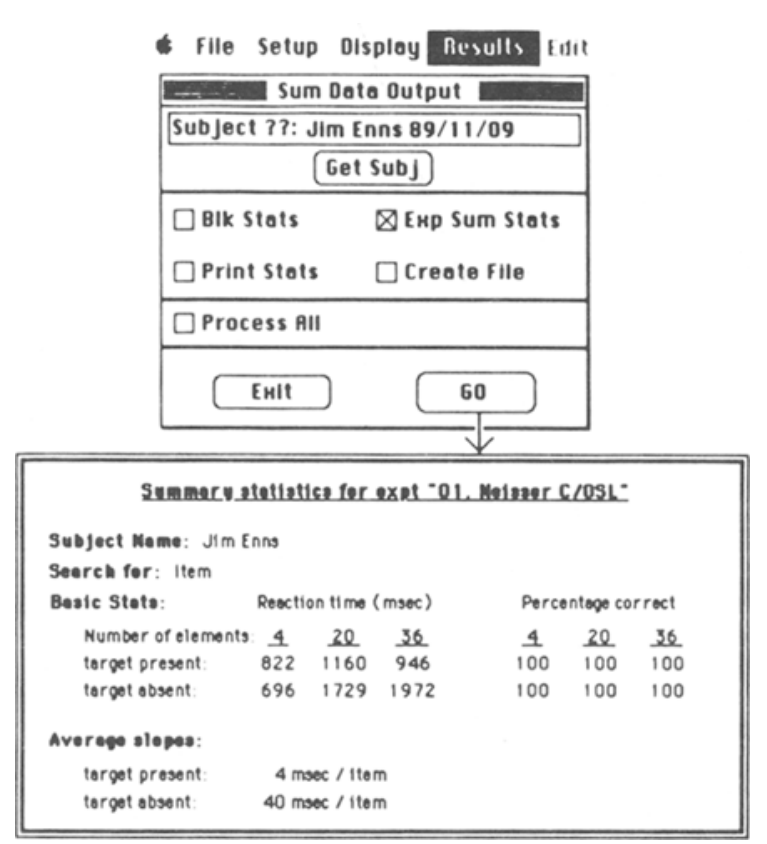

Figure 6. Summary data may be retrieved for examination, for printing, or for storing as files that can later be read by Macintosh statistical packages.

\begin{tabular}{|c|c|}
\hline \multirow{2}{*}{\multicolumn{2}{|c|}{ How Dote Output }} \\
\hline & \\
\hline Clear & Get Subj \\
\hline First BIk 119 & Last BIk 1 (- \\
\hline Х вн IIme & $\otimes \operatorname{Cor} / \operatorname{InCor}(1 / 0)$ \\
\hline QKeyPr P/A(1/0) & $\Delta$ Irial P/A(1/0) \\
\hline QUSI's (scens) & $\otimes \operatorname{Targets}(1,2,3)$ \\
\hline QDistractors & $\otimes D i s p . ~ S \mid z e$ \\
\hline$\square$ Pint Header & $\otimes$ Number Trials \\
\hline$\square$ Printer & Q Create Flle \\
\hline Enit & 60 \\
\hline
\end{tabular}

Figure 7. The raw data can be stored for access by a statistical package or printed for examination. All the information associated with each trial or only those variables that are explicitly selected by the user may be saved and/or printed.

Treisman claimed that the "pop out," or parallel search result, was diagnostic of the fundamental features of early vision.

Search for feature conjunctions (Treisman \& Gelade, 1980). For other items (e.g., T vs. L; red X vs. red O and green $X)$, search time increases linearly with increases in distractor number, and the slopes of target-absent functions are twice those of target-present functions. Treisman believes that these results are diagnostic of search for the conjunction of simple features. Search for feature conjunctions requires the operation of attention to glue the simple features together.

When items are chosen so that they represent highly discriminable values along some dimension, search for feature conjunctions can sometimes become parallel (Treisman, 1988; Wolfe, Franzel, \& Cave, 1988). A striking example of this is parallel search for large, dark Os against a background of large, light Xs, small, dark Xs, and small, light Os. This result is taken as evidence that it is possible for subjects to inhibit visual representations of some dimensions.

Search asymmetry (Treisman \& Gormican, 1988; Treisman \& Souther, 1985). It is very easy to search for a target defined by the presence of a simple feature, but it is much more difficult to search for a target defined by the absence of the same feature. Many dimensions are now known to exhibit this asymmetry in search rate. Although theories differ in their accounts of this effect, it is possible to summarize the results by noting that it is easier to look for "more in less" than to look for "less in more."

Search for three-dimensional features (Enns \& Rensink, 1990). With respect to features, it is important to distinguish between the world of three-dimensional objects (i.e., the scene) and the projection of light from the scene onto a two-dimensional array (i.e., the image). The relations between simple image features "pop out" when they correspond to scene properties (e.g., direction of lighting, three-dimensional orientation), but they require serial search otherwise.

\section{Future Improvements to VSEARCH and IMAKER}

At present, VSEARCH (Version 2.0, November 1989) supports the following variations on the visual search paradigm:

Pre- and Postdisplay masking. Masks are often used in conjunction with brief display exposures, to erase the visible persistence of the image from the decaying phosphor of the screen and from the iconic storage mechanism in the visual system. Mask items used to tile the screen before and/or after the display presentation are constructed and placed into VSEARCH in exactly the same way as target and distractor items.

Variable exposure durations. Up to eight different intervals can be specified for each of the events in the stimulus sequence: preparation time, premask, blank, display, blank, postmask, feedback. This permits response times and accuracy to be studied as a function of display, mask, or blank interval duration.

Deadline procedures. Up to eight intervals can be specified between the display and an auditory tone, which can be used as a signal for the subject to respond (Wickelgren, 1977). This procedure permits the entire range of the speed-accuracy tradeoff to be examined for a given set of items and/or search conditions.

Search for regions of items. A user-defined region of items may be specified as the target to be searched for 
against a background of distractor items. Region size can be varied (similar to varying display size in item-search mode), and it is possible to present up to three targetregion elements and three distractor-region elements. These can be either mixed together in the same display or presented individually in separate trials.

At present, VSEARCH does not support the following variations on the search paradigm, although each of these extensions is at one of various stages of completion toward future installments:

Predisplay cuing. Knowledge about the target identity or location is given before the display is onset. Such experiments can be used to map out the time course of attentional activation (Posner, 1980), the velocity of attention movements (Tsal, 1983), and the effect of target knowledge on search rates (Treisman, 1988).

Postdisplay cuing. Target reports follow an identity or location cue that appears following the stimulus display. These experiments permit examination of iconic persistence (Sperling, 1960) and short-term memory search (Sternberg, 1967).

Multiple simultaneous targets. The number of targets is varied in addition to the total number of items. These experiments allow for fine-grained testing of alternative quantitative processing models.

Circular displays. All items are presented on an imaginary circle centered on the fixation point. These experiments permit the effects of retinal eccentricity to be either controlled or studied systematically (Duncan \& Humphreys, 1989).

More than two alternative responses. This capability would be useful for designing target location tasks and multiple response class experiments.

Classification experiments. Many stimuli (more than three) are mapped onto the same response class.

Color displays. Visual displays are limited at present to two levels of gray (i.e., black/white), allowing VSEARCH to run on all members of the Macintosh family. Extensions to full-color (i.e., 256 gray levels or 256 hues) are planned, but that program will be limited to the Mac II and subsequent Macintoshes that support color (e.g., the IIx and SE/30).

Motion displays. Target items could be defined as a sequence of items in a given location, rather than as simply the present static image. This would permit the investigation of properties of motion comparable to studies of form and color.

\section{Conclusion}

VSEARCH and IMAKER provide an effective way to carry out a wide variety of visual search experiments on a popular family of personal computers. Our hope is that a significant portion of the vision research community will find it useful, thus promoting the rapid acquisition and analysis of visual search data, the easy replication and exchange of experiments between labs, and the flexible adap- tation and extension of existing ideas in visual search to new domains.

\section{Availability}

A VSEARCH DEMO disk that illustrates all features (with the exception that it does not permit the storing or printing of data) is available at no cost, while the complete VSEARCH package (Version 2.0, November 1989) is available for $\$ 200$ US ( $\$ 250$ Canadian) from the first author.

\section{REFERENCES}

BECK, J. (1982). Textural segmentation. In J. Beck (Ed.), Organization and representation in perception (pp. 285-317). Hillsdale, NJ: Erlbaum.

DUNCAN, J., \& HumphreYs, G. W. (1989). Resemblance theory of visual search. Psychological Review, 96, 433-458.

ENNS, J. T., \& RENSINK, R. (1990). Scene-based features influence visual search. Science, 247, 721-723.

Gibson, E. J., \& LeVIN, H. (1975). The psychology of reading. Cambridge, MA: MIT Press.

JulEsZ, B. (1981). Textons, the elements of texture perception, and their interactions. Nature, 290, 91-97.

NeISSER, U. (1963). Decision time without reaction-time: Experiments in visual scanning. American Journal of Psychology, 76, 376385 .

NeIsSer, U. (1964). Visual search. Scientific American, 210, 94-102.

Neisser, U. (1967). Cognitive psychology. Englewood Cliffs, NJ: Prentice-Hall.

Posner, M. I. (1980). Orienting of attention. Quarterly Journal of Experimental Psychology, 32, 3-25.

RENSINK, R. (1990). Toolbox-based routines for Macintosh timing and display. Behavior Research Methods, Instruments, \& Computers, 22, 105-117.

SChNeIDER, W., \& SHIFfrin, R. M. (1977). Controlled and automatic human information processing: I. Detection, search, and attention. Psychological Review, 84, 1-66.

SPERLING, G. (1960). The information available in brief visual presentations. Psychological Monographs, 74 (No. 11).

STERNBERG, S. (1967). Two operations in character recognition: Some evidence from reaction-time measurements. Perception \& Psychophysics, 2, 45-53.

Treisman, A. (1988). Features and objects: The fourteenth Bartlett Memorial Lecture. Quarterly Journal of Experimental Psychology, 40A, 201-237.

Treisman, A., \& Gelade, G. (1980). A feature integration theory of attention. Cognitive Psychology, 12, 97-136.

Treisman, A., \& Gormican, S. (1988). Feature analysis in early vision: Evidence from search asymmetries. Psychological Review, 95 , $15-48$.

Treisman, A., \& Souther, J. (1985). Search asymmetry: A diagnostic for preattentive processing of separable features. Joumal of $E x$ perimental Psychology: General, 114, 285-310.

Tsal, Y. (1983). Movements of attention across the visual field. Journal of Experimental Psychology: Human Perception \& Performance, 9, 523-530.

Tsotsos, J. (1988). A 'complexity level' analysis of immediate vision. International Journal of Computer Vision, 1, 303-320.

WiCKELGREN, W. A. (1977). Speed-accuracy tradeoff and information processing dynamics. Acta Psychologia, 41, 67-85.

Wolfe, J. M., Franzel, S. L., \& Cave, K. R. (1988). Parallel visual search for conjunctions of color and form. Journal of the Optical Society of America, 4, 95.

Zucker, S. (1987). Early vision. In S. Shapiro (Ed.), Encyclopedia of artificial intelligence (pp. 1131-1152). New York: Wiley. 